\title{
Air temperature measurements based on the speed of sound to compensate long distance interferometric measurements
}

\author{
Milena Astrua ${ }^{1, a}$, Marco Pisani ${ }^{1}$ and Massimo Zucco ${ }^{1}$ \\ ${ }^{1}$ INRiM, strada delle Cacce 73 - 10135 Torino - Italy
}

\begin{abstract}
A method to measure the real time temperature distribution along an interferometer path based on the propagation of acoustic waves is presented. It exploits the high sensitivity of the speed of sound in air to the air temperature. In particular, it takes advantage of a special set-up where the generation of the acoustic waves is synchronous with the amplitude modulation of a laser source. A photodetector converts the laser light to an electronic signal considered as reference, while the incoming acoustic waves are focused on a microphone and generate a second signal. In this condition, the phase difference between the two signals substantially depends on the temperature of the air volume interposed between the sources and the receivers. The comparison with the traditional temperature sensors highlighted the limit of the latter in case of fast temperature variations and the advantage of a measurement integrated along the optical path instead of a sampling measurement. The capability of the acoustic method to compensate the interferometric distance measurements due to air temperature variations has been demonstrated for distances up to $27 \mathrm{~m}$.
\end{abstract}

\section{Aim of the research}

One of the main problems in interferometric measurements is the contribution of air refractive index. Generally, it can be calculated from the measured environmental parameters such as temperature, ambient pressure and relative humidity on the basis of mathematical models such as the Edlen's formula [1-3] or other alternative formulae proposed by Ciddor or by Bonsch and Potulski [4,5]. A relative accuracy of the order of $10^{-7}$ on the refractive index (which means an accuracy of $0.1{ }^{\circ} \mathrm{C}$ on temperature) is quite easily achievable for short distances in controlled environment, while in case of measurement of long distances this level of accuracy is still a challenge [6-8].

At INRiM a facility for interferometric measurement of long distances is operating. It is based on a relative heterodyne interferometer, with a moving arm made by a retro-reflector mounted on a carriage sliding on a rail 28 $\mathrm{m}$ long [9]. It aims at the calibration of absolute interferometers and telemeters at a level of uncertainty of about few parts in $10^{-7}$, or it can be used to calibrate laser distance measuring instruments (EDM). In order to ascertain the INRiM calibration and measurement capabilities in this field, we recently participated to the EURAMET project n. 1169 [10], just focused on the comparison of EDMs.

The temperature of the laboratory is roughly controlled at $20^{\circ} \mathrm{C}$ by the air-conditioning plant and the real temperature distribution is measured by 14 thermistors along the interferometric path, at intervals of $2 \mathrm{~m}$. The system works well for slow temperature variations, but it is based on sampling and it is not effective when rapid air temperature changes occurs because of the thermometers time constant. Thus, in order to measure the real time temperature distribution along the interferometer path, a method based on the propagation of acoustic waves has been developed, exploiting the fact that the acoustic waves are about 2 thousands times more sensitive to air temperature variation than the electromagnetic waves. The principle has already been used by Korpelainen [11] for distances of about $5 \mathrm{~m}$.

The set-up and the measuring method together with the comparison with the local temperature measurements are described in this paper. The effectiveness of the acoustic method to correct fast temperature changes effects on interferometric measurements is finally demonstrated.

\section{The method}

In the acoustic system developed at INRiM and drawn in fig. 1, a sinusoidal wave with frequency $f$ in the ultrasound range is sent to a loudspeaker while a digital signal synchronous with the first is used to drive a laser source close and parallel to the loudspeaker. The laser is thus switched on and off synchronously with the generation of the acoustic wave.

In front of them, at a distance variable up to $27 \mathrm{~m}$, the amplitude modulated laser light is received by a

\footnotetext{
${ }^{\mathrm{a}}$ Corresponding author: m.astrua@inrim.it
} 
photodetector and converted to an electronic signal, considered as reference; next to the photodetector, the incoming acoustic waves are focused on a microphone placed in the focus of a parabolic reflector and an electronic circuit extracts a sinusoidal signal at frequency $f$. The phase difference $\Phi$ between the two signals depends on the distance between the transmitter and the receiver, $d$, on the acoustic frequency, $f$, and on the speed of sound in air, $c_{0}$, according to the simple equation: $\Phi=d \cdot f / c_{0}$. In turn, the speed of sound in air strictly depends on the air temperature and on the other environmental parameters, through a rather complicated formula reported in references $[12,13]$.

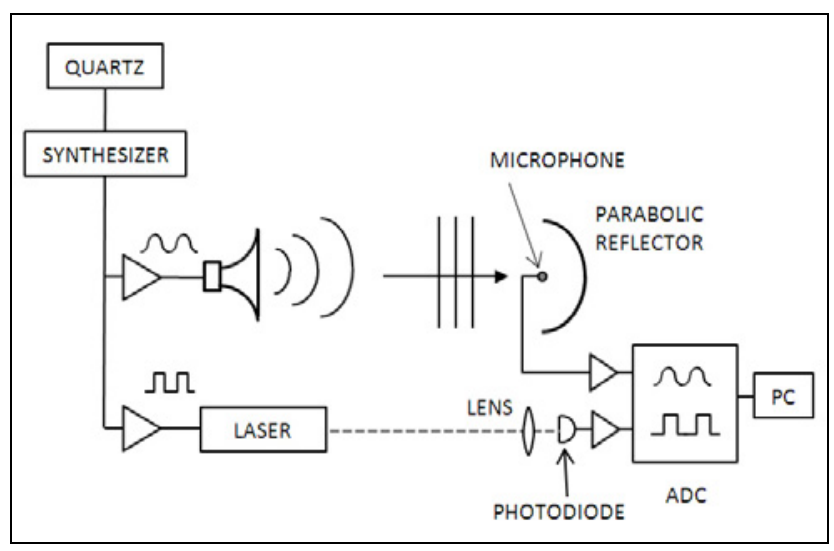

Figure 1. Electronic circuit.

In order to measure the air temperature variations that affect the interferometer path, the loudspeaker and the laser source are mounted next to the retroreflector on board of the carriage and parallel to the optical interferometer axis, as shown in fig. 2.

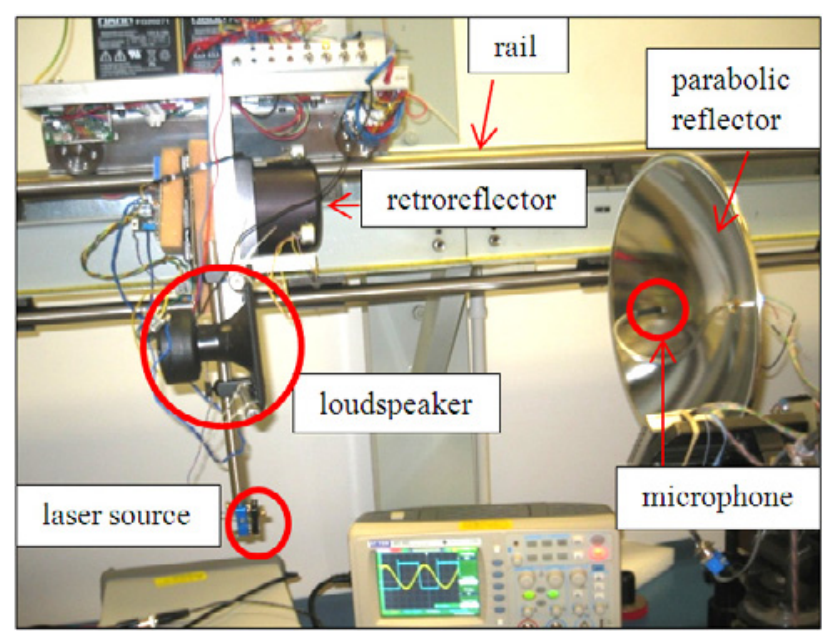

Figure 2. Photograph of the experimental set-up, when the carriage is at the beginning of the rail, close to the parabolic reflector: on the left, the carriage with the optical retroreflector, the loudspeaker and the laser source is visible; on the right the parabolic mirror to focus the acoustic waves on the microphone is visible; the photodetector is not visible since it is hidden behind the mirror.

In front of them at the beginning of the rail, the photodetector and the microphone are placed: the microphone is mounted in the focus of a parabolic reflector which stands on a tripod, while the photodetector is fixed to the interferometer board.

The signals coming from the photodetector and from the microphone are sent to a data acquisition device (NI6259) with a maximum sampling rate of $500 \mathrm{kSamples} / \mathrm{s}$ and a program implemented in LabView ${ }^{\circledR}$ is used to calculate the phase difference between the two signals with an Inphase Quadrature (IQ) demodulation. The same acquisition system is used to acquire the signals coming from the interferometer, so that the interferometer displacement is recorded synchronously with the speed of sound measurement at a rate up to 5 points per second.

The air temperature variations measured by means of the acoustic method will be compared to the ones measured by the 14 thermistors, $30 \mathrm{kOhm}$ type, uniformly dislocated along the rail and automatically recorded during the measurement process by means of a switch unit (Agilent 34970A), at a rate of a complete scan of all the thermistors every 7 seconds. The experimental set-up is sketched in figure 3 .

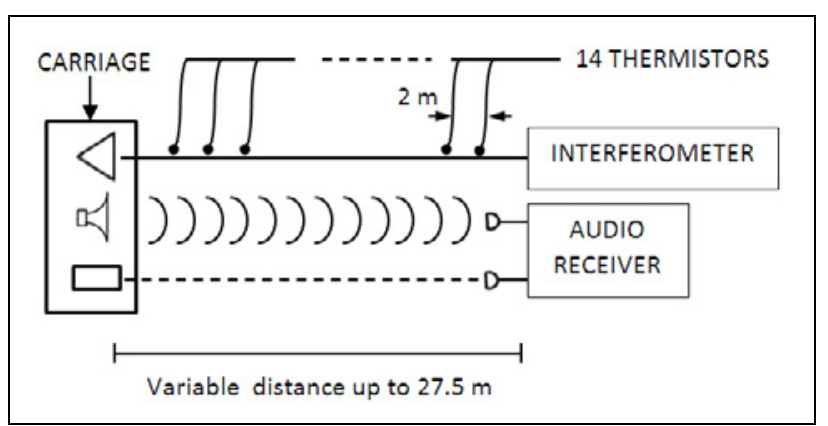

Figure 3. Experimental set-up: on the left, the carriage with the optical retroreflector, the loudspeaker and the laser source is outlined; on the right, the optical interferometer and the audio receiver system are represented. 14 thermistors are dislocated along the optical path, one every $2 \mathrm{~m}$.

\section{Results}

\subsection{Comparison with thermistors}

In order to compare the acoustic method with the local temperature measurements performed by means of the thermistors, a cyclic air temperature modulation was induced acting on the temperature conditioning plant of the laboratory.

The comparison between the two air temperature measurement techniques is reported in Fig 4 and 5.

In fig 4 , the temperature variation is quite slow (a period of about 3 hours) and the agreement between the acoustic method and the averaged thermistor measurement is really good. Very different are the results reported in fig 5 , where the temperature variations were much faster than in the previous case (a period of 10 minutes). On this temporal scale, it is evident that the acoustic method is faster than the thermistors in the response to the air temperature variations, even though the thermistors are extremely little so they were expected to be fast enough to follow the temperature variations. 


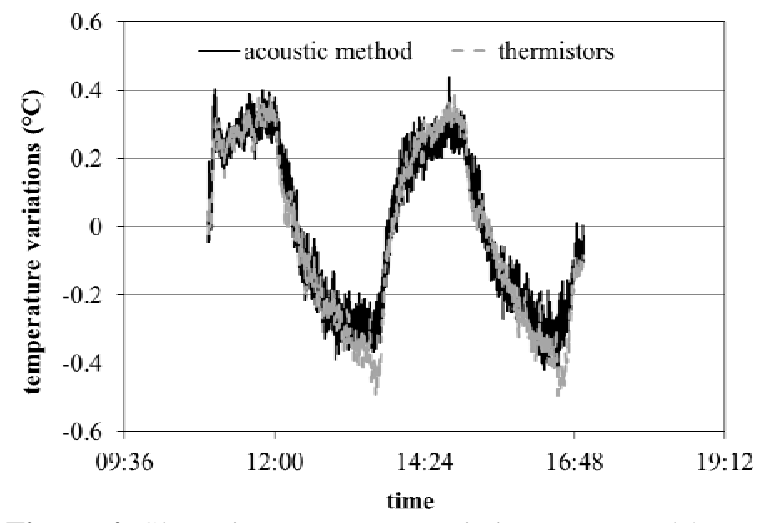

Figure 4. Slow air temperature variations measured by means of the acoustic method and by the thermistors.

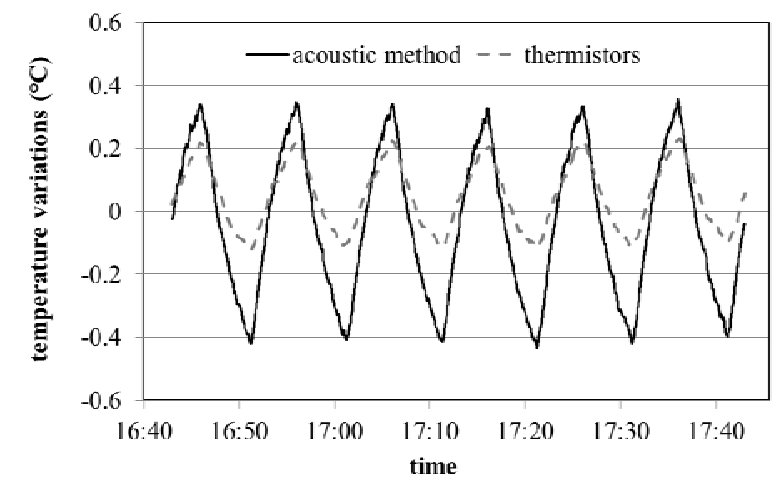

Figure 5. Fast air temperature variations measured by means of the acoustic method and by the thermistors.

\subsection{Uncertainty evaluation}

The traceability of the measurements reported in fig 4 is given by the calibration of the 14 thermometers with respect to a reference platinum resistor in the interval 18$22{ }^{\circ} \mathrm{C}$; the calibration uncertainty is $0.1^{\circ} \mathrm{C}(\mathrm{k}=2)$. As for the acoustic thermometer is not easy to establish a comprehensive uncertainty budget. The main influence parameters are the frequency stability and the sensitivity of the phase measurement including electronics noise, spurious acoustic noise and phase meter resolution. Considering the frequency fluctuations negligible (quartz stability is better than $10^{-6}$ ) we have estimated the measurement noise on the basis of experimental data, i.e. we have calculated the standard deviation of a typical acquisition run in condition of constant temperature (checked with the thermistors). The result gives us a standard deviation of the phase measurement converted in temperature of $0.007{ }^{\circ} \mathrm{C}$ over 1 hour acquisition when the temperature stability measured with the thermometers was $0.004{ }^{\circ} \mathrm{C}$. The comparison results are compliant with the above uncertainty estimation, nevertheless we have to consider that the major contribution to the comparison uncertainty is that the air temperature sampling was not ideal (one thermometer every $2 \mathrm{~m}$ ) and that thermometers have a not negligible integration time. That explains the slight difference between the two curves in fig 4 when the conditioning plant is switched on and hot air starts to flow. A better experiment should be implemented with a larger number of thermometers along the interferometer optical path.

\subsection{Application to the interferometric measurements}

In order to evaluate the capability of the acoustic method to compensate the air temperature variations on the interferometric distance measurement, the signal from the interferometer was synchronously registered with the speed of sound measurement, with the carriage at rest at a distance of $27 \mathrm{~m}$. The air temperature was modulated according to a cycle of approximately 10 minutes acting on the temperature conditioning plant of the laboratory.

Fig 6 shows the changes in the interferometric measurement (solid grey curve) induced by the air temperature variations: when the temperature grows, the optical length measured by the interferometer apparently decreases because of the decreasing of the refractive index, as explained in the introduction. When we correct the distance measured by the interferometer for the air temperature variations measured by the acoustic system (solid black curve) the refractive index effect is cancelled. The residual changes in interferometric measurements (about 5-6 $\mu \mathrm{m}$ peak-to-peak) are the real physical length changes of the rail supporting the interferometer induced by the thermal effect on the whole structure. This result indeed is compliant with the variations of the rail length estimated on the basis of the temperature directly measured on the rail. The dashed grey curve instead represents the interferometric measurement corrected with the temperature measured with the thermometers. Because of the long-time constant of the thermometers the fast variations are not fully corrected.

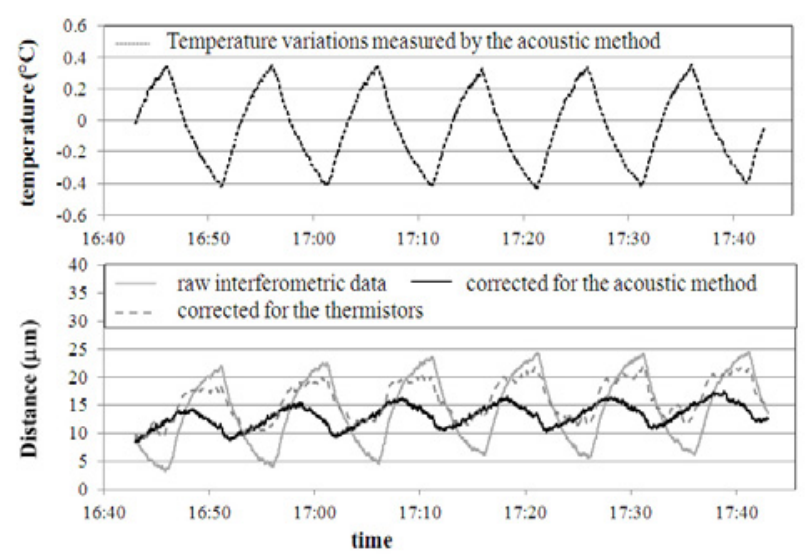

Figure 6. Effect of air temperature variations on the interferometric distance measurement.

\section{Conclusions}

An acoustic method for refractive index estimation for long distance interferometric measurements in air has been developed. The comparison with the traditional temperature sensors highlighted the advantages of a measurement technique which is able to follow even fast temperature variations and which is integrated along the 
optical path instead of a sampling measurement requiring a high number of sensors. The capability of the acoustic method to compensate the interferometric distance measurements due to air temperature variations has been demonstrated.

\section{References}

1. Edlen B, Metrologia 2, pp 71-80 (1966)

2. Birch K.P. and Downs M.J., Metrologia 30, pp 155-162 (1993)

3. Birch K.P. and Downs M.J., Metrologia 31, pp 315-316 (1994)

4. Ciddor PE, Appl. Opt. 35 (9), pp 1566-1573 (1996)

5. Bonsch G. and Potulski E., Metrologia 35, pp 133-139 (1998)

6. J-P Wallerand et al, Proc. of the 14th International Metrology Congress, Paris (France), June 2009, p. 1-3

7. http://www.ptb.de/emrp/sib60-project.html

8. http://projects.npl.co.uk/luminar/

9. M.Pisani et al., Proc of 15 th International Metrology Congress, Paris (France) October 2011

10. http://kcdb.bipm.org/

11. V. Korpelainen and A. Lassila, Opt. Eng. 43(10), 2400-2409 (October 2004)

12. Cramer O, J. Acoust. Soc. Am. 93 (5), pp 2510-2515 (1993)

13. CEI EN 61094-2:2010-09 